\title{
Spontaneous EEG theta/beta ratio and delta-beta coupling in relation to attentional network functioning and self-reported attentional control
}

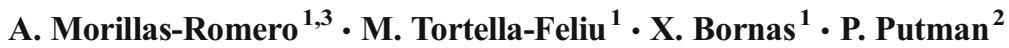

Published online: 10 April 2015

(C) Psychonomic Society, Inc. 2015

\begin{abstract}
Relationships between electroencephalographic (EEG) slow- and fast-wave frequency bands are considered to be of interest in the study of dispositional affective traits, emotion regulation, and attentional phenomena. However, to date, no previous studies had explored whether both state performance-based and self-reported attentional control (AC) measures potentially relate to different patterns of spontaneous EEG measures, in the absence of emotional stimuli. In the present study, individual differences in spontaneous EEG theta/beta ratio and delta-beta coupling at frontal and parietal sites were explored in a sample of 110 healthy volunteers as potential correlates of individual differences in performancebased attentional network functioning, as measured through the Attentional Network Test for Interactions (ANT-I) and self-reported AC. We found that stronger delta-beta coupling at parietal sites was associated with higher self-reported AC. However, no significant associations were found between executive control network functioning and the EEG ratio or coupling measures. Furthermore, a lower spontaneous frontoparietal theta/beta ratio was found to be associated with better orienting network functioning. These results are discussed with a focus on the potential utility of spontaneous EEG measures in several cortical regions for capturing trait-like individual differences in temperament-related factors.
\end{abstract}

A. Morillas-Romero

alfonsomorillas83@gmail.com

1 University Institute for Research on Health Sciences (IUNICS IdISPa), University of the Balearic Islands, Palma, Mallorca, Spain

2 Department of Psychology, Leiden Institute for Brain and Cognition, Leiden University, Leiden, The Netherlands

3 Universitat de les Illes Balears, Edifici IUNICS, Carretera de Valldemossa km.7.5, 07122 Palma, Mallorca, Spain
Keywords Spontaneous EEG $\cdot$ Theta/beta ratio $\cdot$ Delta-beta coupling $\cdot$ Attentional network functioning $\cdot$ Attentional control

Relationships between spontaneous electroencephalographic (EEG) slow-wave (SW; i.e., the theta 4-7 Hz, delta $1-3 \mathrm{~Hz}$ bands) and fast-wave (FW; i.e., the beta $13-30 \mathrm{~Hz}$ band) frequency band activity may reflect the cortical-subcortical interactions involved in affective processes (Knyazev, 2007) and are considered to be of interest in the study of emotional states, dispositional affective traits, emotion regulation, and related processes and capabilities (e.g., Arns, Conners, \& Kraemer, 2013; Massar, Kenemans, \& Schutter, 2014; Putman, Arias-Garcia, Pantazi, \& van Schie, 2012; Schutter \& Knyazev, 2012; Schutter \& Van Honk, 2005a; van Peer, Roelofs, \& Spinhoven, 2008; Velikova et al., 2010).

Two often-used EEG indices are the SW/FW ratio and SW-FW coupling. The SW/FW ratio results from dividing the SW power density (i.e., theta) by the FW power density (i.e., beta) for each individual participant's EEG recording at a selected brain site or selected sites, with higher ratio scores reflecting relatively more SW than FW power. It has been postulated that a relative predominance of SW over FW power may reflect reduced cortical control function over subcortical drives related to motivational imbalances (Schutter \& Van Honk, 2005a). On the other hand, SW-FW coupling (usually delta-beta coupling) is operationalized as the positive correlation between SW and FW in a selected sample, which usually compares samples that have scored high or low on a criterion variable (e.g., trait anxiety; see Putman et al., 2012). In general terms, a positive correlation between delta and beta power is thought to reflect stronger functional cortical-subcortical cross-talk associated with emotion regulation processes (e.g., Schutter \& Van Honk, 2005b). 
Several studies have reported relationships between these spontaneous EEG spectrum parameters and attentional phenomena such as inhibitory control functioning and attentional bias to threat (e.g., Putman, 2011; Putman et al., 2012; Putman, van Peer, Maimari, \& van der Werff, 2010). Putman et al. (2010) reported that the theta/beta ratio at bilateral frontal sites was inversely correlated with fearful modulation of response inhibition in an emotional go/no-go task (i.e., inhibitory control functioning) and with self-reported attentional control (AC). Another study by the same group replicated the negative relationship between theta/beta ratio and selfreported AC, and also showed that the baseline theta/beta ratio predicted acute changes in self-reported $\mathrm{AC}$ in response to stress (Putman, Verkuil, Arias-Garcia, Pantazi, \& van Schie, 2014). Moreover, increased SW/FW ratio has been robustly associated with incentive-motivated disadvantageous decision-making (Schutter \& Van Honk, 2005a) and with psychological disorders characterized by lower attentional control capabilities and reduced inhibitory control, such as ADHD (see Arns et al., 2013, for a review).

Regarding coupling measures, spontaneous reduced frontal delta-beta coupling has been associated with anxiety-related disorders such as obsessive-compulsive disorder (Velikova et al., 2010) and trait anxiety (Putman et al., 2012), and also with increased attentional avoidance of threat (Putman, 2011) and higher interference effects during an emotional Stroop task (Putman et al., 2012). By contrast, SW-FW coupling measures have not been found to be significantly related to self-reported AC (Putman et al., 2012), even though these researchers did expect to find a positive relationship between delta-beta coupling and self-reported AC.

All of the aforementioned studies with spontaneous EEG SW/FW measures had employed some emotionally laden tasks for assessing attentional-inhibitory control functioning. To our knowledge, no previous studies have addressed the question of whether these spontaneous EEG parameters could also be related to state performance-based AC when facing neutral (nonemotionally laden) stimuli and without previous emotional induction. We consider this issue an interesting one to explore because it is related to the intriguing question of whether AC difficulties are specific to emotionally relevant stimuli or may reflect a much broader dysregulation of AC, even when threat-related stimuli are absent. Some theoretical accounts with considerable empirical support, such as the attentional control theory (Eysenck \& Derakshan, 2011; Eysenck, Derakshan, Santos, \& Calvo, 2007), have posited that high trait anxiety impairs the efficiency of two executive functions that involve attentional control (attentional inhibition and attentional shifting). However, there is still controversy as to whether the executive control deficits associated with trait anxiety or negative affect are global, regardless of the emotional value of the stimuli, or whether they concern specific deficits in employing inhibitory processes only when facing emotional stimuli (e.g., Bishop, 2009; Cohen, Daches, Mor, \& Henik, 2014; De Lissnyder, Koster, Derakshan, \& De Raedt, 2010).

On that basis, the primary aims of the present study were (a) to simultaneously examine how spontaneous frontoparietal EEG theta/beta ratio and delta-beta coupling, independently from trait anxiety, might relate to attentional executive control network functioning; (b) to replicate previous findings reporting that the theta/beta ratio is related to trait self-reported AC; and (c) to explore whether delta-beta coupling is also related to trait self-reported AC.

Although, in overall terms, AC has been equated with executive functioning (e.g., Derryberry \& Reed, 2002; Eysenck et al., 2007), research has reported inconsistent results when analyzing the relationships between state performance-based $\mathrm{AC}$ measures (i.e., state $\mathrm{AC}$ ) and trait self-reported $\mathrm{AC}$ (e.g., Muris, van der Heiden, \& Rassin, 2008; Pacheco-Unguetti, Acosta, Marqués, \& Lupiáñez, 2011; Reinholdt-Dunne, Mogg, \& Bradley, 2009; Reinholdt-Dunne, Mogg, \& Bradley, 2013). Some authors have speculated that self-reported AC is akin to perceived AC and not a "true" reflection of attentional capabilities, because high negative affectivity could determine individuals' perceptions of their impaired attentional capabilities, beyond any actual impairment (Tortella-Feliu et al., 2014). In this sense, the study of potential physiological correlates that are associated with both state and trait AC, such as EEG SW/FW ratio and coupling measures, could shed light on the topic. Moreover, we decided also to include EEG recordings of parietal areas, in addition to recordings at frontal electrodes, because the parietal cortex has also been recognized as playing a relevant role in attentional and executive control functioning (e.g., Balle et al., 2013; Kanai, Dong, Bahrami, \& Rees, 2011; Mevorach, Humphreys, \& Shalev, 2006; Mevorach, Humphreys, \& Shalev, 2009; MorillasRomero, Tortella-Feliu, Bornas, \& Aguayo-Siquier, 2013; Posner, Rueda, \& Kanske, 2007).

We hypothesized that both a lower resting fronto-parietal theta/beta ratio and higher resting fronto-parietal delta-beta coupling would be related to more efficient functioning of the attentional executive control network (i.e., less interference, better state performance-based AC), independently from trait anxiety. It was also expected that self-reported trait AC would be negatively associated with theta/beta ratio and, following the theoretical rationale proposed by Putman et al. (2012), positively related to delta-beta coupling. Furthermore, it was expected that the above-mentioned associations would be apparent in frontal and parietal locations.

Beyond the main aim of the study, we also aimed to explore for the first time the potential relationships between the resting fronto-parietal EEG theta/beta ratio or delta-beta coupling and functioning of the attentional orienting and alerting networks in neutral conditions. Because no empirical evidence exists, our testing of these relationships was markedly exploratory. 


\section{Method}

\section{Participants}

A total of 135 healthy, unselected university students and staff members (99 females, 36 males; $M_{\text {age }}=29.60$ years, $S D=$ 10.19 , range 18-55) participated and provided informed consent. Participants were recruited via electronic or posted advertisements and also took part in a larger study (see MorillasRomero, Tortella-Feliu, Balle, \& Bornas, 2014; Tortella-Feliu et al., 2014). One participant was excluded due to missing self-reported data, and two were excluded because of extreme underperformance on the attentional task (accuracy $<90 \%$ in all blocks of the task). For the EEG recordings, the data from 22 participants were excluded due to bad-quality recordings (e.g., hardware failure, excessive movement). The global sample was $n=110$ participants ( 83 females, 27 males; $M_{\text {age }}=$ 29.34 years, $S D=10.17$, range $18-55$ ). For our $\mathrm{SW} / \mathrm{FW}$ ratio analyses, three participants were excluded due to extreme ratio values (more than three standard deviations from the sample mean), and the sample retained for analysis was thus $n=107$ participants ( 80 females, 27 males; $M_{\text {age }}=29.23$ years, $S D=$ 10.22). For SW-FW coupling, the data of two participants exhibiting extreme values in delta or beta powers were discarded, resulting in a sample of $n=108$ participants (83 females, 25 males; $M_{\text {age }}=29.28$ years, $S D=10.20$ ).

\section{Material and procedure}

Self-reports on attentional control capabilities and trait anxiety were collected through an online version of the trait questionnaires before participants were invited to attend a laboratory session. The average time elapsed between the online questionnaire session and the laboratory appointment was 2.25 days $(S D=0.49)$. Once in the laboratory, a resting electrophysiological baseline from each participant was recorded previous to performance of the Attentional Network Test for Interactions (ANT-I; Callejas, Lupiáñez, \& Tudela, 2004). Electrophysiological recordings were conducted individually in a dimly lit and sound-attenuated room. Participants were seated in a comfortable chair in front of a 20-in. computer monitor. After a 3-min adaptation period, EEG activity was recorded during an 8-min resting baseline: 2 min eyes opened, 2 min eyes closed, 2 min eyes opened, and 2 min eyes closed. Then, after a 10-min break, participants completed the ANT-I task. They were asked to refrain from alcohol, drug use, and caffeinated beverages for $4 \mathrm{~h}$ prior to attending the laboratory. The University Bioethics Committee approved all procedures.

\section{Self-reported measures}

Attentional control The Attentional Control Scale (ACS; Derryberry \& Reed, 2002) comprises 20 items to be rated on a 4-point Likert-type scale $(1=$ Almost never, $4=$ Always $)$ measuring the self-reported trait ability to voluntarily control attention, with items pertaining to attentional inhibition and shifting functions and the flexibility of cognitive control. For our sample, the internal consistency was $\alpha=.822$.

Trait anxiety The State-Trait Anxiety Inventory, Trait subscale (STAI-T; Spielberger, Gorsuch, \& Lushene, 1970), was used. It consists of 20 statements measuring subjective feelings of anxiety on a 4-point Likert-type scale ( 1 = Almost never, 4 = Almost always). The internal consistency of our sample was $\alpha=.937$.

\section{EEG data recording and processing}

EEG measures were acquired using a Brain Vision amplifier (Brain Products, Munich, Germany). Spontaneous EEG activity was recorded using a Lycra stretch cap (Easycap) from the F3, F4, C3, C4, P3, P4, T7, T8, O1, and O2 positions, placed in accordance with the International 10/20 System and using electronically coupled mastoid electrodes as the reference channels. EEG signals were offline-segmented in epochs of 512 data points $(1,024 \mathrm{~ms})$ and filtered online with a bandpass filter $(0.05-40 \mathrm{~Hz})$. A $50-\mathrm{Hz}$ notch filter was also applied. The sampling rate was set at $500 \mathrm{~Hz}$, and electrode impedances were kept below $10 \mathrm{k} \Omega$. An electrooculogram (EOG) channel was recorded using two electrodes placed $2 \mathrm{~cm}$ above and below the right eye, and ocular correction (Gratton, Coles, \& Donchin, 1983) was applied using a blink detection algorithm taking the EOG channel as the reference. Segments containing residual muscle movements or other forms of artifacts greater than $100 \mu \mathrm{V}$ were rejected automatically prior to further analysis. From each of the four periods of $120 \mathrm{~s}$ that was recorded (eyes opened, eyes closed, eyes opened, and eyes closed), we took the EEG signal from 30 to $90 \mathrm{~s}$ - that is, each signal was $60 \mathrm{~s}$ (30,000 data points) long. Following Putman et al. (2010), all eyes-open and eyes-closed segments were collapsed for the analysis, and a composite measure averaging EEG power values was computed. A fast Fourier transformation (FFT) with a $10 \%$ Hamming window length was used to estimate the spectral power density $\left(\mu \mathrm{V}^{2} / \mathrm{Hz}\right)$ for the frontal (F3, F4) and parietal (P3, P4) electrodes in the delta (1-3 Hz), theta $(4-7 \mathrm{~Hz})$, and beta $(13-30 \mathrm{~Hz})$ frequency bands. A single bilateral coupling estimate for the frontal and parietal sites was obtained by averaging the power mean activity $\left(\mu \mathrm{V}^{2} / \mathrm{Hz}\right)$ of the two frontal (i.e., F3, F4) and two parietal (i.e., P3, P4) leads.

Individual theta/beta ratio values were calculated by dividing the SW power density (i.e., theta) by the FW power density (i.e., beta) at frontal and parietal sites. Ratio values were log-normalized in order to correct for significantly positively skewed distributions. For the coupling analyses, sub- and supramedian group delta-beta coupling values (see below 
for details) were calculated through Pearson's partial correlations, controlling for age and trait anxiety between the SW power density (i.e., delta) and the FW power density (i.e., beta) at frontal and parietal sites. Power mean values were log-normalized in order to correct for significantly positively skewed distributions.

As we stated in the introduction, trait anxiety has to be taken into account due to its potential detrimental effects on $\mathrm{AC}$ capabilities, and anxiety has previously been reported to be related to delta-beta coupling and AC. Age has also been found to be related to individual differences in attentional network functioning (Zhou, Fan, Lee, Wang, \& Wang, 2011). If any, gender differences in both performance-based and self-reported measures, as well in the EEG indices, would also be taken into account throughout the analyses.

\section{Attentional Network Test for Interactions (Callejas et al., 2004)}

The ANT-I is a modified version of the Attentional Network Test (Fan, McCandliss, Sommer, Raz, \& Posner, 2002), designed to assess the functioning of three major attentional networks - alerting, orienting, and executive control-as was proposed in one of the most influential models of attention as a group of systems (see Posner et al., 2007, for a review). The ANT-I also allows for analysis of the interactions within these networks (for a detailed explanation of the present task, please see Tortella-Feliu et al., 2014).

\section{Statistical analyses}

For the theta/beta ratio analyses, partial correlations controlling for age and trait anxiety (as well as for gender, where appropriate) were conducted for analyzing the associations between theta/beta ratio and the $\mathrm{AC}$ measures. For the coupling analyses, partial correlations controlling for age and trait anxiety (as well as for gender, where appropriate) between delta and beta power at both frontal and parietal sites served as our operationalization of coupling. In order to compare frontal and parietal EEG coupling between participants with low versus high scores on state performance-based attentional measures (i.e., ANT-I) and trait self-reported AC, median split groups were created for each variable. Participants who scored exactly the median values were excluded from the analyses. Fisher's $r$-to- $z$ transformation was used to compare the coupling values between the low- and high-score groups.

For both the ratio and coupling analyses, ANT-I measures were log-normalized in order to correct for significantly positively skewed distributions. For each bivariate correlation analyzed, the Mahalanobis distance (MD) test was conducted in order to explore for the presence of potential bivariate outliers $(t>10.59, p<.005)$. Further results for each correlation will be presented without including these cases. Alpha was set at
.05 , two-sided, but for some strong hypothesis-driven analyses (directional hypotheses regarding the relationships between SW/FW and performance-based or self-reported AC), the criterion was set at a one-sided alpha value of .05. All analyses were conducted using SPSS 15.0 for Windows.

\section{Results}

\section{Descriptive statistics and preliminary analyses}

In a preliminary analysis, we examined reaction times (RTs) in the ANT-I for accurate trials by means of a mixed factorial analysis of variance (ANOVA) of the overall intrasubject effects. Following previous studies (e.g., Pacheco-Unguetti et al., 2011), extreme values (faster than 200 and slower than $1,200 \mathrm{~ms}$ ) were eliminated, in order to avoid anticipations and very long response latencies, respectively. Each one of the 110 participants performed 192 trials in the ANT-I task, for a total of 21,120 trials $(110 \times 192)$. The average number of trials discarded per participant due to the RT $(<200$ or $>1,200 \mathrm{~ms})$ was $3.09(1.61 \%)$. The RTs for response trials were introduced as a dependent variable into a 2 (Alertness: no alerting, alerting tone) $\times 3$ (Orienting: valid, invalid, uncued) $\times 2($ Interference: congruent, incongruent) factorial mixed ANOVA to explore the overall attentional effects.

Consistent with previous studies, the main effects of alertness, orienting, and interference, as well as the interactions between attentional networks, were all statistically significant, showing the pattern usually observed in this task (e.g., Callejas et al., 2004; Pacheco-Unguetti et al., 2011).

Descriptive statistics and gender differences for the attentional-network-functioning derived indices, selfreported AC, trait anxiety, and spontaneous EEG measures are depicted in Table 1.

As is shown in the table, significant gender differences appeared for the executive control and orienting networks. Specifically, women showed greater interference effects (i.e., lower executive control) and greater orienting functioning than men. No significant differences were found for selfreported AC or trait anxiety. Regarding the spontaneous EEG measures, women exhibited significantly greater parietal delta power, as well as greater beta power at both frontal and parietal sites, than did men. Considering these differences, subsequent analyses related to attentional capabilities and spontaneous EEG indices were conducted while controlling for gender in addition to age and trait anxiety.

\section{Theta/beta ratio, ANT-I measures, self-reported AC, and trait anxiety}

The partial correlations, controlling for gender, age, and trait anxiety, between frontal (and parietal) theta/beta ratio and 
Table 1 Descriptive statistics and gender comparisons for ANT-I networks indices, self-report measures, and spontaneous power EEG mean activity (in $\left.\mu \mathrm{V}^{2} / \mathrm{Hz}\right)(n=110)$

\begin{tabular}{|c|c|c|c|c|c|c|c|c|c|c|c|c|}
\hline & \multicolumn{3}{|l|}{ Total } & \multicolumn{2}{|l|}{ Men } & \multicolumn{2}{|l|}{ Women } & \multirow[b]{2}{*}{$t$} & \multirow[b]{2}{*}{$d f$} & \multirow[b]{2}{*}{$p$} & \multirow[b]{2}{*}{ Diff. 95 \% CI } & \multirow[b]{2}{*}{ Cohen's $d$} \\
\hline & $M$ & $S D$ & Median & $M$ & $S D$ & $M$ & $S D$ & & & & & \\
\hline \multicolumn{13}{|l|}{ ANT-I Networks } \\
\hline Interference & 91.74 & 25.14 & 90.66 & 82.63 & 15.76 & 94.70 & 26.93 & -2.84 & 76.85 & .006 & {$[-20.50,-3.62]$} & -0.635 \\
\hline Orienting & 57.98 & 21.21 & 59.87 & 46.12 & 21.10 & 61.84 & 19.88 & -3.51 & 108 & .001 & {$[-24.57,-6.85]$} & -0.784 \\
\hline Alertness & 42.37 & 24.13 & 37.26 & 37.52 & 26.10 & 43.95 & 23.41 & -1.20 & 108 & .231 & {$[-17.00,4.15]$} & -0.268 \\
\hline \multicolumn{13}{|l|}{ Self-Report Measures } \\
\hline Attentional control & 54.20 & 8.84 & 53 & 55.11 & 7.60 & 53.91 & 9.23 & 0.60 & 108 & .544 & {$[-2.70,5.09]$} & 0.134 \\
\hline Trait anxiety & 22.30 & 11.59 & 20 & 22.44 & 11.48 & 22.25 & 11.69 & 0.07 & 108 & .941 & {$[-4.92,5.30]$} & 0.015 \\
\hline \multicolumn{13}{|c|}{ Spontaneous EEG Measures } \\
\hline Frontal delta & 1.777 & 0.778 & 1.695 & 1.539 & 0.700 & 1.854 & 0.700 & -1.85 & 108 & .067 & {$[-0.65,0.02]$} & -0.413 \\
\hline Frontal theta & 0.414 & 0.208 & 0.356 & 0.369 & 0.237 & 0.429 & 0.197 & -1.29 & 108 & .139 & {$[-0.15,0.03]$} & -0.288 \\
\hline Frontal beta & 0.098 & 0.070 & 0.082 & 0.070 & 0.040 & 0.107 & 0.076 & -2.40 & 108 & .018 & {$[-0.06,-0.006]$} & -0.536 \\
\hline Parietal delta & 1.499 & 0.630 & 1.341 & 1.221 & 0.662 & 1.589 & 0.596 & -2.71 & 108 & .008 & {$[-0.63,-0.09]$} & -0.605 \\
\hline Parietal theta & 0.378 & 0.200 & 0.338 & 0.329 & 0.204 & 0.395 & 0.197 & -1.49 & 108 & .139 & {$[-0.15,-0.02]$} & -0.333 \\
\hline Parietal beta & 0.085 & 0.047 & 0.075 & 0.066 & 0.041 & 0.091 & 0.047 & -2.53 & 108 & .013 & {$[-0.04,-0.006]$} & -0.565 \\
\hline
\end{tabular}

Delta $=1-3 \mathrm{~Hz}$, theta $=4-7 \mathrm{~Hz}$, beta $=13-30 \mathrm{~Hz}$; Frontal = Average of power mean activity $\left(\right.$ in $\left.\mu \mathrm{V}^{2} / \mathrm{Hz}\right)$ at F3 and F4; Parietal = Average of power mean activity (in $\mu \mathrm{V}^{2} / \mathrm{Hz}$ ) at $\mathrm{P} 3$ and $\mathrm{P} 4$

both attentional network functioning and self-reported $\mathrm{AC}$ are depicted in Table 2.

No significant associations appeared between theta/beta ratio and executive control functioning at either frontal or parietal sites. However, as is shown in the table, theta/beta ratios at both frontal and parietal sites were significantly negatively associated with orienting network functioning. Specifically, a greater presence of fast waves relative to slow ones at frontal and parietal sites was associated with higher orienting capabilities. On the contrary, alertness network functioning was not significantly related with theta/beta ratio at any brain sites.

Regarding self-reported AC, no significant relationships were found between theta/beta ratio and self-reported $\mathrm{AC}$ at either frontal or parietal sites.

Table 2 Partial correlations (with $p$ values in parentheses) controlling for gender, age and trait anxiety between frontal and parietal theta/beta ratio and both performance-based and self-reported measures of attentional control

\begin{tabular}{lll}
\hline & \multicolumn{2}{l}{ Theta/Beta Ratio } \\
\cline { 2 - 3 } & Frontal & Parietal \\
\hline Executive network (i.e. Interference) & $-.089\left(.188^{\mathrm{a}}\right)$ & $.083\left(.202^{\mathrm{a}}\right)$ \\
Orienting network & $-.217\left(.030^{\mathrm{b}}\right)$ & $-.279\left(.005^{\mathrm{b}}\right)$ \\
Alertness network & $-.090\left(.752^{\mathrm{b}}\right)$ & $-.116\left(.244^{\mathrm{b}}\right)$ \\
Self-reported attentional control & $-.117\left(.240^{\mathrm{a}}\right)$ & $-.043\left(.332^{\mathrm{a}}\right)$ \\
\hline
\end{tabular}

a One-sided $p$ value

b Two-sided $p$ value

\section{Delta-beta coupling, ANT-I measures, self-reported AC, and trait anxiety}

Regarding performance-based attentional network functioning, no significant coupling differences were found between high (above-median) and low (below-median) scorers for any of the attentional networks (i.e., executive control, orienting, and alertness) at either frontal or parietal sites (see Table 3 ).

As for self-reported AC, no significant coupling differences were found between high and low scorers groups at frontal sites. However, in parietal areas, participants who reported higher self-reported AC exhibited higher delta-beta coupling values than did their counterparts showing lower self-reported AC.

\section{Discussion}

The present study was mainly devoted to exploring whether two different spontaneous EEG measures, the SW/FW ratio and SW-FW coupling, were associated with both state performance-based AC and trait self-reported AC. Secondly, in a more exploratory fashion, we also examined whether these spontaneous EEG measures were associated with the functioning of the orienting and alertness attentional networks. Furthermore, to the best of our knowledge, this has been the first study to report on whether these spontaneous EEG measures were related to attentional network functioning 
Table 3 Delta-beta coupling comparisons between low and high scorers (median split) for each study variable at both frontal and parietal sites

\begin{tabular}{|c|c|c|c|c|c|c|c|c|}
\hline & \multicolumn{4}{|l|}{ Frontal } & \multicolumn{4}{|l|}{ Parietal } \\
\hline & Delta-Beta Coupling & $n$ & $z$ & $p$ & Delta-Beta Coupling & $n$ & $z$ & $p$ \\
\hline \multicolumn{9}{|c|}{ Executive Network (i.e., Interference) } \\
\hline Low & .059 & 54 & & & .434 & 54 & & \\
\hline High & .016 & 53 & 0.22 & $.412^{\mathrm{a}}$ & .227 & 54 & 1.21 & $.113^{\mathrm{a}}$ \\
\hline \multicolumn{9}{|c|}{ Orienting Network } \\
\hline Low & -.133 & 53 & & & .293 & 54 & & \\
\hline High & .222 & 54 & -1.81 & $.070^{\mathrm{b}}$ & .364 & 54 & -0.4 & $.689^{\mathrm{b}}$ \\
\hline \multicolumn{9}{|c|}{ Alertness Network } \\
\hline Low & .206 & 54 & & & .456 & 54 & & \\
\hline High & -.117 & 53 & 1.64 & $.101^{\mathrm{b}}$ & .188 & 54 & 1.52 & $.128^{\mathrm{b}}$ \\
\hline \multicolumn{9}{|c|}{ Self-Reported Attentional Control } \\
\hline Low & -.056 & 47 & & & .186 & 48 & & \\
\hline High & .162 & 53 & -1.06 & $.144^{\mathrm{a}}$ & .506 & 53 & -1.8 & $.035^{\mathrm{a}}$ \\
\hline \multicolumn{9}{|c|}{$\begin{array}{l}\text { Delta-beta coupling }=\text { Partial correlations controlling for age, gender, and trait anxiety between } \mathrm{LN}(\text { delta) power mean activity }(1-3 \mathrm{~Hz}) \text { and LN (beta) } \\
\text { power mean activity }(13-30 \mathrm{~Hz}) \text {; Frontal = Average of power mean activity }\left(\text { in } \mu \mathrm{V}^{2} / \mathrm{Hz}\right) \text { at F3 and F4; Parietal = Average of power mean activity (in } \\
\left.\mu \mathrm{V}^{2} / \mathrm{Hz}\right) \text { at P3 and P4 }\end{array}$} \\
\hline \multicolumn{9}{|c|}{ a One-sided $p$ value } \\
\hline b Two-si & lue & & & & & & & \\
\hline
\end{tabular}

without involving emotional stimuli and/or without previous emotional induction.

Regarding our main aim, surprisingly, none of the expected associations appeared, since neither theta/beta ratio nor deltabeta coupling was significantly associated with state performance-based AC (i.e., executive control network functioning). In this sense, it should be stressed that in the present study, unlike in previous studies that have used emotional stimuli (Putman, 2011; Putman et al., 2012; Putman et al., 2010), the functioning of the attentional executive control network was assessed by means of an attentional task that included only neutral (i.e., non-emotionally-laden) stimuli, without any kind of emotional induction. As we commented, there has been considerable controversy regarding whether the executive control deficits associated with trait anxiety might emerge regardless of the emotional value of the stimuli or, conversely, whether they exclusively arise when facing emotional stimuli (e.g., Bishop, 2009; Cohen et al., 2014; De Lissnyder et al., 2010). From the present results, it could be argued, therefore, that such state AC difficulties are specific only to emotionally relevant stimuli, instead of reflecting a broader dysregulation of AC.

A second potential explanation that could account for the unexpected results in the association between our SW/FW indices and performance-based $\mathrm{AC}$ is related to the type of conflict used to assess the participants' ability to inhibit taskirrelevant distractions. Berggren and Derakshan (2014) have recently reported that only stimulus-response conflict increased task-irrelevant distraction for high-trait-anxiety individuals as compared to their low counterparts, and that group differences did not arise in stimulus-stimulus conflict tasks. Berggren and Derakshan concluded that distinguishing among types of conflict resolution may be important to properly analyze impaired inhibition, especially in individualdifferences approaches. In the flanker task that we used in the present study, conflict probably emanates both from stimulus-response competition (e.g., irrelevant but incongruent arrows flanking the target, and the arising response competition) and from stimulus-stimulus conflict (e.g., distracting irrelevant arrows that simply are not the target one; Berggren \& Derakshan, 2014). Then, we could speculate that with the ANT-I, interference effects are not enough related to stimulusresponse competition to capture individual differences in spontaneous ratio indices.

Regarding self-reported AC, although previous studies had reported a negative relationship between trait self-reported $\mathrm{AC}$ and frontal theta/beta ratio (Putman et al., 2010, Putman et al., 2014), we were unable to replicate this association. However, we found that increased parietal delta-beta coupling was associated with higher self-reported AC. The lack of an association between theta/beta ratio and self-reported AC is somewhat difficult to interpret. However, methodological differences could be potential reasons for this difference in results. For example, the mean age value for our sample was approximately 10 years older than that for Putman and colleagues' participants (Putman et al., 2010; Putman et al., 2014). As we previously commented, the influence of age in attentional capabilities has to be taken into account, since attentional skills 
decrease over the years (e.g., Zhou et al., 2011), and also because self-reported $\mathrm{AC}$ and theta/beta ratio have been related to age (Putman et al., 2010). We may speculate that, although we controlled for variance in age within our sample, the large overall age difference between our sample and those in previous studies might be responsible for this divergence of findings. All in all, failure to replicate past research cannot be considered a discouraging issue by itself, especially when the study has been conducted with a larger and more heterogeneous sample than was used in previous studies. Moreover, previous research on this topic is not very extensive; that is, our results are not contradictory with a well-established fact derived from a substantial amount of research. Our data simply provide evidence that theta/beta ratio is not always related to self-reported AC.

With regard to spontaneous SW-FW coupling measures, as we commented, a significant association appeared between self-reported AC and parietal (but not frontal) delta-beta coupling, with individuals who scored higher on trait selfreported $\mathrm{AC}$ exhibiting greater parietal coupling values than their counterparts with lower self-reported AC. The only prior study devoted to exploring this potential relationship (Putman et al., 2012) did not find any significant association between frontal delta-beta coupling and trait AC, although a positive association was also hypothesized. Since AC has been previously related to effective emotion regulation capabilities (e.g., Rothbart \& Rueda, 2005), and given that increased delta-beta coupling has been postulated to reflect stronger cortical-subcortical communication involved in affect regulation (e.g., Knyazev, 2007; Schutter \& Van Honk, 2005b) as well as with reduced emotional Stroop interference (Putman et al., 2012), it should not be surprising that increased spontaneous delta-beta coupling could be associated with trait AC. It could be argued that delta-beta coupling may reflect top-down processing involved both in cognitive control and in affect regulation. Furthermore, the fact that delta-beta coupling differences related to trait self-reported AC appeared at parietal sites seems to highlight the desirability of also including this brain area when exploring attentional capabilities, as has also been noted in the previous literature (e.g., Balle et al., 2013; Fan et al., 2002; Mevorach et al., 2006; Morillas-Romero et al., 2013; Putman et al., 2014; Tortella-Feliu et al., 2014).

The facts that no significant association was found between SW-FW coupling and performance-based AC, but that an association was found with self-reported $\mathrm{AC}$, raises the question of whether the latter measure reflects perceived AC, and so is more influenced by affective aspects. This could explain its significant association with parietal coupling. Moreover, because self-reported $\mathrm{AC}$ has not been found to be associated with theta/beta ratio, we might speculate that coupling measures perhaps better capture the aforementioned affective component, which deserves to be specifically investigated in future research. In any case, the divergence between the two
$\mathrm{AC}$ measures does not undermine the value of AC self-report by itself, since it can inform research into the affective features that impact beliefs about one's own attentional capabilities.

Furthermore, the association between trait $\mathrm{AC}$ and SWFW coupling only appeared in parietal and not in frontal regions. Since both brain areas are considered to be part of a structural-functional network recruited during attentional functioning (e.g., Corbetta, Patel, \& Shulman, 2008; Posner et al., 2007), a significant degree of temporal coherence in their spontaneous activity might be expected to remain (e.g., Corbetta et al., 2008). The exact reason why no significant association was found for frontal areas remains elusive to us. However, it is worth noting, firstly, that inconsistent findings dominate the literature regarding the role of parietal areas (e.g., Stewart, Towers, Coan, \& Allen, 2011). Secondly, when recording spontaneous EEG oscillations it is impossible to know which cognitive processes - with parietal and/or frontal cortex potentially playing some of their complex roles (see Bisley \& Goldberg, 2010, for a review) - are taking place in participants' minds. Therefore, it is possible that sometimes the EEG dynamics from parietal regions show some associations with trait $\mathrm{AC}$, whereas in other studies these associations concern frontal areas. And thirdly, the likelihood of such seemingly incongruent results is increased when EEG activity is measured at only a few electrodes, as in the present study. In any case, our results do not compromise the potential validity of such cross-talk measures.

In a very exploratory fashion, and taking into account that no previous research has been devoted to this issue, in this study we also intended to examine potential relationships between spontaneous EEG ratio and coupling measures and the functioning of orienting and alertness networks. We found that both lower frontal and parietal theta/beta ratios were related to greater orienting network efficiency. However, no significant associations with delta-beta coupling appeared for this network.

Although the relationship between orienting network functioning and temperament-related factors such as trait $\mathrm{AC}$ is still unclear (Moriya \& Tanno, 2009; Tull, Maack, Viana, \& Gratz, 2012), recent studies have reported positive relationships between trait self-reported AC and orienting network functioning (Tortella-Feliu et al., 2014). Thus, it makes sense that the efficiency of the orienting network appeared to be related in the same conceptual direction to self-reported AC since, although no significant association was found in the present study, prior research had shown that $\mathrm{AC}$ was related to a lower theta/beta ratio (Putman et al., 2010; Putman et al., 2014). Furthermore, the fact that participants with lower orienting capabilities showed higher fronto-parietal theta/ beta ratios in resting conditions seems also to be partially in line with recent models suggesting that increased tonic slow EEG activity (i.e., delta and theta) could reflect unstable regulation of brain arousal, deriving in cognitive deficits visible 
through poorer execution of continuous performance-based tasks (see Arns \& Kenemans, 2014; Hegerl \& Hensch, 2014, for reviews).

Finally, in the present study neither theta/beta ratio nor delta-beta coupling was significantly related to alertness network functioning. Although RT responses were significantly faster in trials in which an alerting tone was present, it could be possible that this observed effect derived from more automatic attentional processes being less sensitive to state anxietyrelated processes in the absence of emotional context, as compared to those demanding greater top-down capabilities (i.e., executive functioning). In any case, since no previous studies exist regarding these potential relationships, it is difficult to us to frame this lack of association with both SW/FW measures.

The present study has a number of limitations. The first of these relates to the fact that midline brain electrodes (i.e., Fz, $\mathrm{Cz}$, and $\mathrm{Pz}$ ) were not included in our initial EEG montage. However, given the common strong correlations between F3/ F4 and midline recordings of delta, theta, and beta power, this was probably of limited influence on our results. Additionally, another limitation may relate to the small array of scalp electrodes - two per coronal zone-applied in the recordings, hampering a clear interpretation of the brain activity's topographic distribution and source information.

Furthermore, it would be interesting to take phasic EEG measures while participants were performing the attentional task, since including phasic EEG measures also might have helped us better interpret some of the results related to state performance-based AC. Future research should clarify the debate regarding tonic versus phasic measures, as it relates to delta-beta coupling and executive control functioning, by simultaneously recording EEG activity in both basal and task performance conditions. Moreover, it would be of special interest to directly compare state AC (i.e., executive control functioning) when facing both neutral and threat-related stimuli. Also using a clearer stimulus-response conflict could facilitate findings of greater individual differences in executive control functioning and/or its spontaneous EEG activity correlates. Finally, given that gender differences were found in both attentional network functioning and spontaneous EEG beta power, it would have been interesting to explore the presented analyses separately in women and men, rather than only controlling for gender in the statistical analyses. Regrettably, the small number of male participants did not allow us to conduct separate analyses.

Nonetheless, and in spite of these limitations, to our knowledge this is the first study that has explored whether AC (both trait self-reported and state performance-based) is related to different patterns of spontaneous EEG measures without involving emotional stimuli. Although we failed to replicate previous findings that had related state performance-based $\mathrm{AC}$ and other executive functions when facing emotional stimuli to different patterns of spontaneous EEG measures, the results of the present study showed that spontaneous parietal delta-beta coupling was related with better self-reported AC capabilities. We also found that orienting network functioning was inversely related to the fronto-parietal theta/beta ratio.

Further research will be needed to clarify the usefulness of spontaneous (i.e., in resting conditions) EEG measures as related to the attentional network functioning in the absence of emotional stimuli. However, the present results seem to highlight the potential utility of some of these spontaneous EEG measures (i.e., delta-beta coupling) when studying individual differences in temperament-related factors, such as selfreported $\mathrm{AC}$.

Author note This research was supported by Grant No. PSI200912711 from the Spanish Government. We thank Blanca Aguayo-Siquier and Maria Balle for her assistance with the participants' recruitment and data collection. The present article was mostly written at the University of Leiden (The Netherlands) as a direct benefit derived from the IBROPERC InEurope Short Stay program, supported by the International Brain Research Organization (IBRO).

\section{References}

Arns, M., Conners, C. K., \& Kraemer, H. C. (2013). A decade of EEG theta/beta ratio research in ADHD: A meta-analysis. Journal of Attention Disorders, 17, 374-383. doi:10.1177/1087054712460087

Arns, M., \& Kenemans, J. L. (2014). Neurofeedback in ADHD and insomnia: Vigilance stabilization through sleep spindles and circadian networks. Neuroscience \& Biobehavioral Reviews, 44, 183194. doi:10.1016/j.neubiorev.2012.10.006

Balle, M., Bornas, X., Tortella-Feliu, M., Llabrés, J., Morillas-Romero, A., Aguayo-Siquier, B., \& Gelabert, J. M. (2013). Resting parietal EEG asymmetry and cardiac vagal tone predict attentional control. Biological Psychology, 93, 257-261. doi:10.1016/j.biopsycho. 2013.02.012

Berggren, N., \& Derakshan, N. (2014). Inhibitory deficits in trait anxiety: Increased stimulus-based or response-based interference? Psychonomic Bulletin \& Review, 21, 1339-1345. doi:10.3758/ s13423-014-0611-8

Bishop, S. J. (2009). Trait anxiety and impoverished prefrontal control of attention. Nature Neuroscience, 12, 92-98. doi:10.1038/nn.2242

Bisley, J. W., \& Goldberg, M. E. (2010). Attention, intention, and priority in the parietal lobe. Annual Review of Neuroscience, 33, 1-21. doi: 10.1146/annurev-neuro-060909-152823

Callejas, A., Lupiáñez, J., \& Tudela, P. (2004). The three attentional networks: On their independence and interactions. Brain and Cognition, 54, 225-227. doi:10.1016/j.bandc.2004.02.012

Cohen, N., Daches, S., Mor, N., \& Henik, A. (2014). Inhibition of negative content-A shared process in rumination and reappraisal. Frontiers in Psychology, 5, 622. doi:10.3389/fpsyg.2014.00622

Corbetta, M., Patel, G., \& Shulman, G. L. (2008). The reorienting system of the human brain: From environment to theory of mind. Neuron, 58, 306-324. doi:10.1016/j.neuron.2008.04.017

De Lissnyder, E., Koster, E. H. W., Derakshan, N., \& De Raedt, R. (2010). The association between depressive symptoms and executive control impairments in response to emotional and nonemotional information. Cognition and Emotion, 24, 264-280. doi: $10.1080 / 02699930903378354$ 
Derryberry, D., \& Reed, M. A. (2002). Anxiety-related attentional biases and their regulation by attentional control. Journal of Abnormal Psychology, 111, 225-236. doi:10.1037/0021-843X.111.2.225

Eysenck, M. W., \& Derakshan, N. (2011). New perspectives in attentional control theory. Personality and Individual Differences, 50, 955-960. doi:10.1016/j.paid.2010.08.019

Eysenck, M. W., Derakshan, N., Santos, R., \& Calvo, M. G. (2007). Anxiety and cognitive performance: Attentional control theory. Emotion, 7, 336-353. doi:10.1037/1528-3542.7.2.336

Fan, J., McCandliss, B., Sommer, T., Raz, A., \& Posner, M. (2002). Testing the efficiency and independence of attentional networks. Journal of Cognitive Neuroscience, 14, 340-347. doi:10.1162/ 089892902317361886

Gratton, G., Coles, M. G. H., \& Donchin, E. (1983). A new method for off-line removal of ocular artifact. Electroencephalography and Clinical Neurophysiology, 55, 468-484. doi:10.1016/00134694(83)90135-9

Hegerl, U., \& Hensch, T. (2014). The vigilance regulation model of affective disorders and ADHD. Neuroscience \& Biobehavioral Reviews, 44, 45-57. doi:10.1016/j.neubiorev.2012.10.008

Kanai, R., Dong, M. Y., Bahrami, B., \& Rees, G. (2011). Distractibility in daily life is reflected in the structure and function of human parietal cortex. Journal of Neuroscience, 31, 6620-6626. doi:10.1523/ JNEUROSCI. 5864-10.2011

Knyazev, G. G. (2007). Motivation, emotion, and their inhibitory control mirrored in brain oscillations. Neuroscience \& Biobehavioral Reviews, 31, 377-395. doi:10.1016/j.neubiorev.2006.10.004

Massar, S. A. A., Kenemans, J. L., \& Schutter, D. J. L. G. (2014). Resting-state EEG theta activity and risk learning: Sensitivity to reward or punishment? International Journal of Psychophysiology, 91, 172-177. doi:10.1016/j.ijpsycho.2013.10.013

Mevorach, C., Humphreys, G. W., \& Shalev, L. (2006). Opposite biases in salience-based selection for the left and right posterior parietal cortex. Nature Neuroscience, 9, 740-742. doi:10.1038/nn1709

Mevorach, C., Humphreys, G. W., \& Shalev, L. (2009). Reflexive and preparatory selection and suppression of salient information in the right and left posterior parietal cortex. Journal of Cognitive Neuroscience, 21, 1204-1214. doi:10.1162/jocn.2009.21088

Morillas-Romero, A., Tortella-Feliu, M., Balle, M., \& Bornas, X. (2014). Spontaneous emotion regulation and attentional control. Emotion. Advance online publication. doi:10.1037/emo0000016

Morillas-Romero, A., Tortella-Feliu, M., Bornas, X., \& Aguayo-Siquier, B. (2013). Resting parietal electroencephalogram asymmetries and self-reported attentional control. Clinical EEG and Neuroscience, 44, 188-192. doi:10.1177/1550059412465871

Moriya, J., \& Tanno, Y. (2009). Dysfunction of attentional networks for non-emotional processing in negative affect. Cognition and Emotion, 23, 1090-1105. doi:10.1080/02699930802335018

Muris, P., van der Heiden, S., \& Rassin, E. (2008). Disgust sensitivity and psychopathological symptoms in non-clinical children. Journal of Behavior Therapy and Experimental Psychiatry, 39, 133-146. doi: 10.1016/j.jbtep.2007.02.001

Pacheco-Unguetti, A. P., Acosta, A., Marqués, E., \& Lupiáñez, J. (2011). Alterations of the attentional networks in patients with anxiety disorders. Journal of Anxiety Disorders, 25, 888-895. doi:10.1016/j. janxdis.2011.04.010

Posner, M. I., Rueda, M. R., \& Kanske, P. (2007). Probing the mechanisms of attention. In J. T. Cacioppo, J. G. Tassiary, \& G. G. Berntson (Eds.), Handbook of psychophysiology (pp. 410-431). Cambridge, UK: Cambridge University Press.

Putman, P. (2011). Resting state EEG delta-beta coherence in relation to anxiety, behavioral inhibition, and selective attentional processing of threatening stimuli. International Journal of Psychophysiology, 80, 63-68. doi:10.1016/j.ijpsycho.2011.01.011
Putman, P., Arias-Garcia, E., Pantazi, I., \& van Schie, C. (2012). Emotional Stroop interference for threatening words is related to reduced EEG delta-beta coupling and low attentional control. International Journal of Psychophysiology, 84, 194-200. doi:10. 1016/j.ijpsycho.2012.02.006

Putman, P., van Peer, J., Maimari, I., \& van der Werff, S. (2010). EEG theta/beta ratio in relation to fear-modulated response-inhibition, attentional control, and affective traits. Biological Psychology, 83, 73-78. doi:10.1016/j.biopsycho.2009.10.008

Putman, P., Verkuil, B., Arias-Garcia, E., Pantazi, I., \& van Schie, C. (2014). EEG theta/beta ratio as a potential biomarker for attentional control and resilience against deleterious effects of stress on attention. Cognitive, Affective, \& Behavioral Neuroscience, 14, 782-791. doi:10.3758/s13415-013-0238-7

Reinholdt-Dunne, M. L., Mogg, K., \& Bradley, B. P. (2009). Effects of anxiety and attention control on processing pictorial and linguistic emotional information. Behaviour Research and Therapy, 47, 410 417. doi:10.1016/j.brat.2009.01.012

Reinholdt-Dunne, M. L., Mogg, K., \& Bradley, B. P. (2013). Attention control: Relationships between self-report and behavioural measures, and symptoms of anxiety and depression. Cognition and Emotion, 27, 430-440. doi:10.1080/02699931.2012.715081

Rothbart, M. K., \& Rueda, M. R. (2005). The development of effortful control. In U. Mayr, E. Awh, \& K. Keele (Eds.), Developing individuality in the human brain: A tribute to Michael I. Posner (pp. 167-188). Washington, DC: American Psychological Association.

Schutter, D. J., \& Knyazev, G. G. (2012). Cross-frequency coupling of brain oscillations in studying motivation and emotion. Motivation and Emotion, 36, 46-54. doi:10.1007/s11031-011-9237-6

Schutter, D. J. L. G., \& Van Honk, J. (2005a). Electrophysiological ratio markers for the balance between reward and punishment. Cognitive Brain Research, 24, 685-690. doi:10.1016/j.cogbrainres.2005.04. 002

Schutter, D. J. L. G., \& Van Honk, J. (2005b). Salivary cortisol levels and the coupling of midfrontal delta-beta oscillations. International Journal of Psychophysiology, 55, 127-129. doi:10.1016/j.ijpsycho. 2004.07.003

Spielberger, C. D., Gorsuch, R. L., \& Lushene, R. E. (1970). State-trait anxiety inventory. Palo Alto, CA: Consulting Psychologists Press.

Stewart, J. L., Towers, D. N., Coan, J. A., \& Allen, J. J. (2011). The oftneglected role of parietal EEG asymmetry and risk for major depressive disorder. Psychophysiology, 48, 82-95. doi:10.1111/j.14698986.2010.01035.x

Tortella-Feliu, M., Morillas-Romero, A., Balle, M., Bornas, X., Llabrés, J., \& Pacheco-Unguetti, A. P. (2014). Attentional control, attentional network functioning, and emotion regulation styles. Cognition and Emotion, 8, 769-780. doi:10.1080/02699931.2013.860889

Tull, M. T., Maack, D. J., Viana, A. G., \& Gratz, K. L. (2012). Behavioral inhibition and attentional network functioning. Cognitive Behaviour Therapy, 41, 1-4. doi:10.1080/16506073.2011.614273

van Peer, J. M., Roelofs, K., \& Spinhoven, P. (2008). Cortisol administration enhances the coupling of midfrontal delta and beta oscillations. International Journal of Psychophysiology, 67, 144-150. doi: 10.1016/j.ijpsycho.2007.11.001

Velikova, S., Locatelli, M., Insacco, C., Smeraldi, E., Comi, G., \& Leocani, L. (2010). Dysfunctional brain circuitry in obsessive-compulsive disorder: Source and coherence analysis of EEG rhythms. NeuroImage, 49, 977-983. doi:10.1016/j.neuroimage.2009.08.015

Zhou, S. S., Fan, J., Lee, T. M., Wang, C. Q., \& Wang, K. (2011). Agerelated differences in attentional networks of alerting and executive control in young, middle-aged, and older Chinese adults. Brain and Cognition, 75, 205-210. doi:10.1016/j.bandc.2010.12.003 